\title{
Effects of varying the carbohydrate: fat ratio in a hot lunch on postprandial variables in male volunteers
}

\author{
By J. M. M. VAN AMELSVOORT, P. VAN STRATUM, J. H. KRAAL, \\ R. N. LUSSENBURG AND U. M. T. HOUTSMULLER \\ Unilever Research Laboratorium, P.O. Box 114, 3130 AC Vlaardingen, The Netherlands
}

(Received 1 June 1988 - Accepted 22 September 1988)

\begin{abstract}
1. Healthy male volunteers consumed at noon, hot test meals with four different carbohydrate:fat ratios varying between 2.64 and 0.50 , and composed of fried beefsteak, mashed potatoes, French beans, and a dessert of custard with mashed peaches. The energy content of the meals was $40 \%$ of the daily intake of the volunteers, estimated from their individual dietary histories.

2. Before, and at different times after the start of the meal, blood samples were taken and a number of indices of carbohydrate and lipid metabolism were determined in the samples, i.e. glucose, insulin, free fatty acid, free and total glycerol, free and total cholesterol, high-density-lipoprotein (HDL)-cholesterol and low-density-lipoprotein (LDL)-cholesterol.

3. Increasing the carbohydrate:fat ratio resulted in higher postprandial peaks of glucose and insulin. In addition, the peak area under the postprandial glucose curve showed a significant increase. The peak area under the postprandial insulin curve had also increased, indicating that a larger amount of insulin was secreted by the pancreas on increasing the carbohydrate content in the meal. There was no significant correlation between the height of the postprandial peak of blood glucose and the size of the meal.

4. All four meals caused elevated postprandial blood triacylglycerol levels. However, the decline of this elevated level took a much longer time after the meals with the lower carbohydrate:fat ratios, i.e. containing larger amounts of triacylglycerols. There was a significant decreasing linear relation between the carbohydrate content of the meals and the peak area under the postprandial triacylglycerol curve. Free glycerol and free fatty acids showed lower postprandial levels in the blood after the meals with the higher carbohydrate:fat ratios, and the peak areas of the postprandial curves of both variables displayed a significant decrease. Little or no effect of the meal carbohydrate:fat ratio was observed on the postprandial concentrations of total cholesterol, unesterified cholesterol, HDL-cholesterol or LDL-cholesterol.
\end{abstract}

Dietary habits may affect health by inducing gradual changes in the long term (chronic effects) and by changes occurring after each meal (postprandial effects): the fluctuations induced by meals in blood glucose, blood insulin and blood lipids are increasingly accepted as being relevant in the development of various diseases, e.g. atherosclerosis, obesity, maturity onset diabetes. Therefore scientific interest in the effects of meal composition on postprandial blood variables is growing and the number of studies of postprandial effects is increasing considerably.

Jenkins et al. (1983, 1984a, b, 1985, 1986) have been looking for ways of controlling the rate of nutrient absorption in the management of diabetes and hyperlipidaemia. They are using the concept of the glycaemic index in order to classify the glucose responses in the blood induced by the consumption of various types of food or food ingredients (Wolever $\&$ Jenkins, 1986). However, the glycaemic index may not be the only relevant variable. It has been stressed that it should be extended to include also the measurement of the insulin secretory response at the very least. Indeed, the postprandial insulin response may vary considerably in normal healthy subjects, depending on the type of food in which carbohydrates are incorporated (Shively et al. 1986). In addition, it has been stated that it would be more appropriate to study high-carbohydrate foods when included in meals, than to determine the glycaemic index of individual compounds (Calle-Pascual et al. 1986).

In his study of the postprandial indices of lipid metabolism, Zilversmit $(1979,1984)$ suggested the possibility of postprandial chylomicron remnants playing a part in 
atherogenesis. The most important conclusion reached at a recent workshop at Bethesda, 1-3 July 1986, on the impact of dietary cholesterol on plasma lipoproteins and atherogenesis was that since the human body is in a fasting state for only about $6 \mathrm{~h} / \mathrm{d}$, scientific research should also be directed towards the study of the lipid levels in the non-fasting state in order to obtain information more relevant to the generation of the disease (W. Insull $\mathrm{Jr}$, personal communication).

Recently, health councils in various countries in the Western world have advised a reduction in the consumption of dietary fat to approximately $30 \%$ of the total energy intake. Since in practice this will most likely lead to replacing fat in the diet with carbohydrate, the aim of the present work was to study the effects of varying the carbohydrate: fat ratio (C:F) in a hot meal, eaten at noon, on a number of postprandial indices of carbohydrate and lipid metabolism in the blood of male volunteers. In order to be able to extrapolate the findings of the present study to day-to-day practice as much as possible, the (male) volunteers who were going to participate in the study were asked to continue to follow their normal, habitual daily activity pattern. In addition, the hot test meals had to be of a nearly common composition and appearance, and would be served at noon inbetween normal working hours. The subsequent taking of a number of blood samples at intervals for the study of the postprandial indices of carbohydrate and lipid metabolism should cause minimal disturbance to the normal activities of each individual. In view of the fact that free-living human beings differ widely in respect of their health factors, habitual physical activities and nutritional behaviour, one may expect a large variation in postprandial responses. Therefore, it was decided to let every person be his own control by giving the four test meals to each individual at (at least) 1-week intervals.

\section{METHODS}

\section{Volunteers}

The recruiting procedure was started by inviting interested employees from Unilever Research Laboratorium Vlaardingen to an introductory meeting. At this meeting, the purpose and scope of the experiment, including its medical consequences, were explained in detail. At the end of the meeting volunteers could give their written consent to participate in the study.

The participants had to meet the following requirements: (1) they should participate freely and voluntarily and without expecting any financial reward, (2) they should preferably be representative of people living in Western society, (3) they should be in good general health (as checked by a physician), be able to continue their habitual daily activities during the experiment, and be in a physiologically stable condition during the experiment (approximately 6 weeks). In view of the hormonal variations occurring in women during the monthly cycle, only male volunteers were admitted.

After the recruitment, twenty-eight volunteers passed the medical examination. In the course of the experiment two volunteers withdrew from the study for reasons unrelated to the experiment. Twenty-six healthy male volunteers completed the study. Their height varied between 1.73 and $1.97 \mathrm{~m}$ (mean $1.82 \mathrm{~m}$ ), their body-weight between 62 and $102 \mathrm{~kg}$ (mean $78 \mathrm{~kg}$ ) and their age between 22 and 59 years (mean 40 years). The body mass index (weight $/$ height $^{2}$; BMI) varied between $19.0 \mathrm{~kg} / \mathrm{m}^{2}$ (very lean) and $27.4 \mathrm{~kg} / \mathrm{m}^{2}$ (mildly obese) (mean $23.6 \mathrm{~kg} / \mathrm{m}^{2}$, indicative of a normal non-obese population).

The average daily food intake of the volunteers was assessed by a dietician using the dietary history procedure (Marr, 1971). The nutrients consumed during an average day and at an average hot meal were calculated using the standard Dutch food composition table (Commissie UCV, 1984). 
Table 1. Compositions of the test meals

\begin{tabular}{ccccc}
\hline Meal code & $\begin{array}{c}\text { Carbohydrate* } \\
\text { (\% energy) }\end{array}$ & $\begin{array}{c}\text { Fat } \dagger \\
\text { (\% energy) }\end{array}$ & Carbohydrate:fat & $\begin{array}{c}\text { Protein } \\
\text { (\% energy) }\end{array}$ \\
\hline A & $59 \cdot 3$ & $22 \cdot 5$ & $2 \cdot 64$ & $18 \cdot 2$ \\
B & $48 \cdot 6$ & $33 \cdot 6$ & $1 \cdot 45$ & $17 \cdot 7$ \\
C & $38 \cdot 0$ & $44 \cdot 2$ & 0.86 & $17 \cdot 7$ \\
D & $27 \cdot 3$ & $54 \cdot 7$ & 0.50 & $18 \cdot 0$ \\
\hline
\end{tabular}

* In the carbohydrate fraction the ratio of polysaccharides: (mono+di)saccharides was kept at 2:1.

$\dagger$ The saturated:mono-: polyunsaturated fatty acids ratio was kept at $1: 1: 1$.

\section{Test meals}

The test meals consisted of beefsteak (fried in margarine), mashed potatoes, French beans, and a dessert made with custard and mashed peaches. Four different test meals, coded A, $\mathrm{B}, \mathrm{C}$ and $\mathrm{D}$ with a protein content of approximately $18 \%$ energy and C:F of $2.64,1.45$, 0.86 and 0.50 respectively were used (Table 1 ). The dietary fat contained equal proportions of polyunsaturated, monounsaturated and saturated fatty acids. The polysaccharide:(mono + di)saccharide ratio (P:MD ratio) was kept at 2:1.

Every volunteer consumed each of the four test meals, with at least a 1-week interval, on Tuesday, Wednesday or Thursday. As there were four different test meals, they could be offered to the volunteers in twenty-four different orders. As there were twenty-eight volunteers, all twenty-four possible orders were applied, and four of them twice. The order in which a specific volunteer received the meals was determined by randomization with the restriction that on each day of the experiment not more than eight volunteers could take part, two for each type of test meal. This was realized by constructing a 5-week experimental design; in four of these weeks a volunteer consumed one of the test meals, the remaining week being a free week.

\section{Food consumption}

The volunteers were asked to follow more or less the same consumption pattern, and to avoid incidental excessive intakes of alcohol and snacks at parties, etc. during the $2 \mathrm{~d}$ preceding the day on which one of the test meals was to be consumed. On the test day they consumed their habitual breakfast and stopped drinking sugar-containing beverages after 09.30 hours. The test meals were offered to the group of eight volunteers between 11.40 and 12.10 hours at $4 \mathrm{~min}$ intervals. They were allowed to eat the meal within $30 \mathrm{~min}$. The size of a test meal corresponded to $40 \%$ of the mean daily energy intake of each participant as calculated from the dietary history values. During the consumption of the meals the volunteers were allowed to take table salt and fat-free gravy ad lib. After the meal they were allowed to drink only beverages containing little or no sugar.

In order to measure the consumption of table salt and gravy by the volunteers, each participant received with his meal a weighed salt cellar and a gravy-boat. The intakes of extra salt and gravy were calculated by weighing the salt-cellars and gravy-boats after the meal in the absence of the volunteers. They were not aware of this determination. At $4 \mathrm{~h}$ after the start of the meal the volunteers were questioned about their satiety feelings by means of a scoring list very similar to the list used by Krotkiewski (1984).

Blood sampling, preparation and analysis

Blood samples were taken from the volunteers in a sitting posture shortly before (approximately $10 \mathrm{~min}$ ), and $0 \cdot 5,1,2,4$ and $6 \mathrm{~h}$ after the start of the meal. Blood was taken 
by venous puncture and collected into rubber-stoppered tubes at reduced pressure (Venoject system). Two $5 \mathrm{ml}$ samples and one $2 \mathrm{ml}$ sample were taken at each of the times mentioned previously. The $2 \mathrm{ml}$ tube (for the analysis of glucose) contained sodium fluoride and EDTA. The tubes were kept on ice until all samples to be taken at one time-interval had been obtained. This usually took $28 \mathrm{~min}$ or less. The tubes were then immediately processed in one batch.

The tubes containing the $2 \mathrm{ml}$ blood samples were centrifuged for $10 \mathrm{~min}$ at $1000 \mathrm{~g}$ and $4^{\circ}$. The plasma was carefully removed and stored at $-70^{\circ}$ to be analysed for glucose later. Half the tubes containing the $5 \mathrm{ml}$ samples were kept at $37^{\circ}$, and after $30 \mathrm{~min}$ the blood clots were detached from the wall of the tubes with a needle. The tubes were then centrifuged for $10 \mathrm{~min}$ at $1000 \mathrm{~g}$ and $4^{\circ}$; the serum was aspirated and divided into two portions. One portion was stored immediately at $-70^{\circ}$ to be analysed later for free cholesterol and total cholesterol, and for free glycerol (FG) and total glycerol (TG). The other portion was used for the determination of free fatty acid (FFA), high-densitylipoprotein (HDL)-cholesterol and low-density-lipoprotein (LDL)-cholesterol. The other 5 -mi tubes were put in a water-bath at room temperature. After $60 \mathrm{~min}$ the blood clots were detached from the walls of the tubes, and the tubes were centrifuged for $10 \mathrm{~min}$ at $1000 \mathrm{~g}$ and $4^{\circ}$. The supernatant fraction was aspirated and stored in two portions at $-70^{\circ}$ to be analysed for insulin later.

Glucose concentrations in the plasma samples were determined using the Gluco-quant kit (Boehringer Mannheim, Federal Republic of Germany). Insulin was assayed with the Boehringer Enzyme-Test Insulin kit (ELISA); in this assay a polyclonal antibody, raised against insulin, is used. TG was determined using the Boehringer 2-Components Endpoint method kit. FG was determined with the Boehringer Enzymatic UV-method with a potassium hydroxide hydrolysis kit but omitting the KOH-hydrolysis step. FFA was determined in the freshly prepared serum samples by means of the NEFA-C kit from WAKO-Chemicals (Neuss, Federal Republic of Germany). Total cholesterol was determined with the Boehringer CHOD-PAP method kit. Free cholesterol was determined with the Boehringer free cholesterol kit. The HDL and LDL subfractions were isolated from the serums using the precipitation kit from BioMérieux (Charbonnières les Bains, France). Subsequently, the amounts of HDL-cholesterol and LDL-cholesterol were determined as total cholesterol.

\section{Statistical analysis}

As it was not known beforehand which variable would have the best discriminating power to detect the differences (if any) between the meals, the highest of the observed postprandial values was tested as well as the peak area under the postprandial curve. It was subsequently decided to report the peak area results for all variables. In view of the specific shapes of the postprandial curves of insulin and glucose the maximim values for these criteria were also considered. The values were subjected to analysis of covariance to investigate whether there was any relation between the carbohydrate content ( $\%$ energy) and the variable under investigation, taking into account the intrinsic individual levels as well as the values before the start of the meals.

\section{Ethical considerations}

The protocol of the experiment was analysed for its ethical consequences, and approved by an external committee composed of three independent experts in the field. 
Table 2. Mean energy intake and carbohydrate: fat ratio $(C: F)$ in total daily consumption and during the dinner as calculated from dietary histories of the adult male volunteers

(Mean values with their standard errors for twenty-six subjects)

\begin{tabular}{|c|c|c|c|c|c|c|c|}
\hline & \multirow[b]{2}{*}{ Energy (MJ) } & \multirow{2}{*}{$\begin{array}{l}\text { Percentage } \\
\text { of daily } \\
\text { energy intake }\end{array}$} & \multicolumn{4}{|c|}{ Distribution of energy (\%) } & \multirow[b]{2}{*}{$\mathrm{C}: \mathrm{F}$} \\
\hline & & & Protein & Fat & Carbohydrate & Alcohol & \\
\hline \multicolumn{8}{|c|}{ Total consumption } \\
\hline Mean & 11.5 & - & $13 \cdot 7$ & $39 \cdot 4$ & $41 \cdot 4$ & $5 \cdot 5$ & 1.06 \\
\hline SEM & 0.47 & - & 0.29 & $0 \cdot 61$ & 0.85 & 0.80 & 0.032 \\
\hline \multicolumn{8}{|l|}{ Range } \\
\hline Minimum & $6 \cdot 3$ & - & $10 \cdot 5$ & $34 \cdot 1$ & 32.9 & 0.0 & 0.75 \\
\hline Maximum & $16 \cdot 0$ & - & 16.8 & $44 \cdot 4$ & $50-5$ & $15 \cdot 2$ & 1.48 \\
\hline \multicolumn{8}{|c|}{ Dinner } \\
\hline Mean & $4 \cdot 0$ & 35 & 17.9 & $43 \cdot 0$ & $36 \cdot 1$ & $3 \cdot 0$ & 0.86 \\
\hline SEM & 0.23 & $1 \cdot 3$ & 0.51 & 1.01 & 0.96 & 0.89 & 0.040 \\
\hline \multicolumn{8}{|l|}{ Range } \\
\hline Minimum & $2 \cdot 2$ & 22 & $14 \cdot 0$ & $30-5$ & $26 \cdot 6$ & 0.0 & 0.57 \\
\hline Maximum & 6.9 & 56 & $25 \cdot 7$ & $50 \cdot 2$ & $47 \cdot 3$ & 15.8 & 1.35 \\
\hline
\end{tabular}

RESULTS

Composition and appreciation of test meals

The dietary histories of the volunteers (Table 2) showed that the mean daily energy intake varied between 6.3 and $16.0 \mathrm{MJ}$ and averaged $11.5 \mathrm{MJ}$. The mean energy intakes in the form of protein and carbohydrate were 13.7 and $41.4 \%$ energy respectively. The intake of fat was about $39.4 \%$ energy which is very similar to the present fat intake in Western society. The mean intake of alcohol was $5.5 \%$ energy, but in some cases the alcohol intake amounted to $15 \cdot 2 \%$ of the daily energy intake! $\mathrm{C}: \mathrm{F}$ in the diet was about $1 \cdot 06$, which is between the $C: F$ values of meals $B$ and $C$ (see Table 1). The mean energy intake in the hot meal of the volunteers was about $4.0 \mathrm{MJ}$, which corresponded to $35 \%$ of the total daily energy intake. However, this value varied between 22 and $56 \%$. The contributions of protein and fat in the hot meal were higher than those in the total daily intake. This may, at least partly, be due to the consumption of meat at dinner. In addition, because of the concomitant reduction in the amount of carbohydrates, $C: F$ was lower $(0.86$ in the hot meal and 1.06 in the total consumption). The mean energy intake as alcohol during dinner was lower than in the total intake.

The contributions of polysaccharides to the total daily consumption and to the intake at dinner were very similar (22.6 and $23.9 \%$ respectively, Table 3 ). In contrast, the mono- and disaccharide consumption was higher in the total consumption than in the intake at dinner (18.6 and $12.3 \%$ respectively). This resulted in a higher P:MD in the hot meal $(2 \cdot 24)$ compared with that in the total consumption (1.32).

Also the percentages of saturated fat in the total consumption and in that at dinner were very similar (15.7 and $15.5 \%$ respectively). However, since the percentages of polyunsaturated fat were different, the polyunsaturated:saturated fatty acids $(P: S)$ ratio was appreciably lower in the total consumption than in the intake at dinner $(0.64$ and 0.83 respectively). Both ratios are fairly high compared with those routinely found in Western populations. The total daily dietary fibre consumption and the dietary fibre consumption at dinner were 31.4 and $14.1 \mathrm{~g}$ respectively, which indicates that an appreciable amount of fibre was consumed at dinner. 
Table 3. Energy contribution (\%) of (mono + di)saccharides $(M D)$, polysaccharides $(P)$ and saturated fat, $P: M D$, polyunsaturated : saturated fatty acid ratio $(P: S)$ and content of dietary fibre $(g)$ in the total daily consumption and in the hot meal for adult male volunteers

(Mean values with their standard errors for twenty-six subjects)

\begin{tabular}{|c|c|c|c|c|c|c|}
\hline & \multicolumn{3}{|c|}{ Energy (\%) } & \multirow[b]{2}{*}{$P: M D$} & \multirow[b]{2}{*}{$\mathrm{P}: \mathrm{S}$} & \multirow{2}{*}{$\begin{array}{l}\text { Dietary } \\
\text { fibre } \\
\text { (g) }\end{array}$} \\
\hline & MD & $\mathrm{P}$ & Saturated fat & & & \\
\hline \multicolumn{7}{|c|}{ Total consumption } \\
\hline Mean & $18 \cdot 6$ & 22.6 & $15 \cdot 7$ & $1 \cdot 32$ & 0.64 & $31 \cdot 4$ \\
\hline SEM & 0.82 & 0.68 & 0.34 & $0 \cdot 116$ & 0.037 & 1.58 \\
\hline \multicolumn{7}{|l|}{ Range } \\
\hline Minimum & $8 \cdot 3$ & $15 \cdot 6$ & $12 \cdot 0$ & 0.72 & 0.30 & $21 \cdot 7$ \\
\hline Maximum & $26 \cdot 2$ & 31.5 & 20.6 & 3.80 & 0.94 & $54 \cdot 2$ \\
\hline \multicolumn{7}{|c|}{ Dinner } \\
\hline Mean & $12 \cdot 3$ & $23 \cdot 9$ & $15 \cdot 5$ & $2 \cdot 24$ & 0.83 & $14 \cdot 1$ \\
\hline SEM & 0.77 & 0.94 & 0.55 & 0.24 & 0.058 & 0.92 \\
\hline \multicolumn{7}{|l|}{ Range } \\
\hline Minimum & $5 \cdot 1$ & $15 \cdot 0$ & $9 \cdot 3$ & 1.09 & 0.36 & $7 \cdot 0$ \\
\hline Maximum & 19.8 & $34 \cdot 6$ & $19 \cdot 9$ & 6.78 & $1 \cdot 41$ & $25 \cdot 4$ \\
\hline
\end{tabular}

Although the taste and composition of the test meals were appreciated, a number of participants found it difficult to eat the whole amount. This may be due to a number of factors. First, the total energy content of each meal was adjusted to $40 \%$ of the mean daily energy intake of each participant as calculated from his dietary history. This value was higher than the average individual energy consumption at dinner (35\%, Table 2$)$. The larger meal size was deliberately chosen in order to exaggerate the postprandial responses. A second factor which may have played a part was the wide variation in the daily eating patterns of the test subjects, the majority of the volunteers eating less than $40 \%$, and even less than $35 \%$ of their total energy intake at noon. Third, the volume of the isoenergetic meal increased considerably on increasing C:F from the normal 0.86 to 2.64 in meal A (cf. Tables 2 and 1). The increase in the volume of the meal on increasing $C: F$ may thus offer an opportunity to reduce the energy intake in situations such as obesity. Satiation induced by the volume of the meal, however, vanishes quickly after a short digestive period. Therefore, the volunteers were questioned about their satiety feelings $4 \mathrm{~h}$ after the start of the meal by means of a list with a number of specific descriptions from which they were to select the one giving the best approximation of their satiety feelings (Table 4). The scores indicated higher ratings of hunger feelings (chi-square: $P<0.05$ ) after the fat-rich meals (codes $\mathrm{C}$ and $\mathrm{D}$ ) than after the carbohydrate-rich meals (codes $\mathrm{A}$ and $\mathrm{B}$ ). This indicated that satiety feelings at $4 \mathrm{~h}$ after the meal tended to be less after a meal containing much fat and little carbohydrate (and fibre). Whether this was due to a single component in these meals, e.g. amount and type of fat, carbohydrate or fibre, or to a combination of these factors remains to be established.

The increased dryness and reduced palatability of the high-carbohydrate meals lead to an increase in the consumption of the freely available fat-free gravy and table salt during the meal (Table 5). The mean intake of gravy increased from $45 \mathrm{~g}$ to $96 \mathrm{~g}$ on increasing $\mathrm{C}: \mathrm{F}$ from 0.50 to 2.64 (in meals D-A respectively). The mean voluntary intake of table salt increased from 0.31 to $0.55 \mathrm{~g}$. Hence, the mean total intake of sodium from gravy and table salt together increased from 0.31 to $0.60 \mathrm{~g}$. Although the individual intake of table salt varied considerably, this finding may be a warning that $\mathrm{Na}$ intake could increase, at least 
Table 4. Scoring list of appetite* $4 \mathrm{~h}$ after the start of the test meals for adult male volunteers

(Mean values for twenty-six subjects)

\begin{tabular}{lllccc}
\hline \hline & Test meal $\uparrow \ldots$ & \multicolumn{3}{c}{ Number of scores } \\
& A & B & C & D \\
\hline 0 & No appetite & 1 & 1 & 0 & 0 \\
1 & Can eat, don't need to & $9 \ddagger$ & 1 & 2 & 2 \\
2 & Can eat a small item & 8 & $14 \ddagger$ & 9 & 10 \\
3 & Do have appetite, but can wait & 3 & 2 & $6 \ddagger$ & 4 \\
4 & Would like to eat something nice & 1 & 2 & 3 & $6 \ddagger$ \\
5 & Do have appetite, would like to eat something & 2 & 5 & 5 & 1 \\
6 & Would like to eat now & 1 & 1 & 0 & 1 \\
7 & I'm rather hungry & 1 & 0 & 1 & 2 \\
8 & I'm very hungry & 0 & 0 & 0 & 0 \\
\hline
\end{tabular}

A, B, C, D, carbohydrate: fat of $2 \cdot 64,1.45,0.86,0.50$ respectively.

* Volunteers were asked to select the level giving the best approximation of their feelings of satiety.

$\dagger$ For details, see Table 1 and p. 269.

$\ddagger$ The meals with the highest scores at levels $1-4$.

Table 5. Ad lib. intakes ( $g$ ) of gravy and table salt and sodium in gravy + table salt during the meals, and energy contents $(M J)$ of the meals and gravy consumed by the adult male volunteers

(Mean values with their standard errors for twenty-six subjects)

\begin{tabular}{|c|c|c|c|c|c|c|c|c|}
\hline \multirow[t]{2}{*}{$\begin{array}{l}\text { Carbohydrate: fat ... } \\
\text { Test meal... }\end{array}$} & \multicolumn{2}{|c|}{$\begin{array}{c}2 \cdot 64 \\
\mathrm{~A}\end{array}$} & \multicolumn{2}{|c|}{$\begin{array}{c}1.45 \\
\mathrm{~B}\end{array}$} & \multicolumn{2}{|c|}{$\begin{array}{c}0.86 \\
\mathrm{C}\end{array}$} & \multicolumn{2}{|c|}{$\begin{array}{c}0 \cdot 50 \\
D\end{array}$} \\
\hline & Mean & SE & Mean & $\mathrm{SE}$ & Mean & $\mathbf{S E}$ & Mean & $\mathrm{SE}$ \\
\hline Gravy (g) & 96 & $7 \cdot 3$ & 80 & 6.9 & 62 & $4 \cdot 4$ & 45 & $3 \cdot 8$ \\
\hline Gravy (MJ) & 0.121 & 0.0093 & 0.101 & 0.0087 & 0.079 & 0.0056 & 0.058 & $0 \cdot 0048$ \\
\hline Meal (MJ) & 4.6 & 0.20 & $4 \cdot 6$ & $0-20$ & $4 \cdot 6$ & $0 \cdot 20$ & $4 \cdot 6$ & 0.20 \\
\hline Table salt (g) & 0.55 & $0 \cdot 132$ & 0.42 & 0.092 & 0.42 & $0 \cdot 105$ & 0.31 & 0.070 \\
\hline $\begin{array}{l}\text { Sodium in gravy } \\
+ \text { salt }(\mathrm{g})\end{array}$ & 0.60 & 0.061 & 0.49 & $0-044$ & 0.42 & $0 \cdot 040$ & $0 \cdot 31$ & 0.034 \\
\hline
\end{tabular}

in part of the population, when dietary $C: F$ is increased. The intake of gravy also resulted in a small increase in the consumption of metabolizable energy because the mean energy provided by the gravy changed from 0.058 to $0.121 \mathrm{MJ}$. This difference is not likely to induce significant effects on the postprandial variables since the energy from gravy is only a very small part of the total energy consumed $(1 \cdot 3-2 \cdot 6 \%)$.

\section{Blood glucose}

The postprandial curves of blood glucose for the four meals are shown in Fig. 1. The glucose concentrations at the start of the meals did not differ significantly, which indicates that the volunteers had followed more or less the same consumption pattern in the $24-48 \mathrm{~h}$ before each meal. This supports the reliability of any difference found in postprandial blood gluose levels. For all meals the highest mean value in the postprandial curves was observed at $30 \mathrm{~min}$ after the start of the meal. The glucose concentration at the 


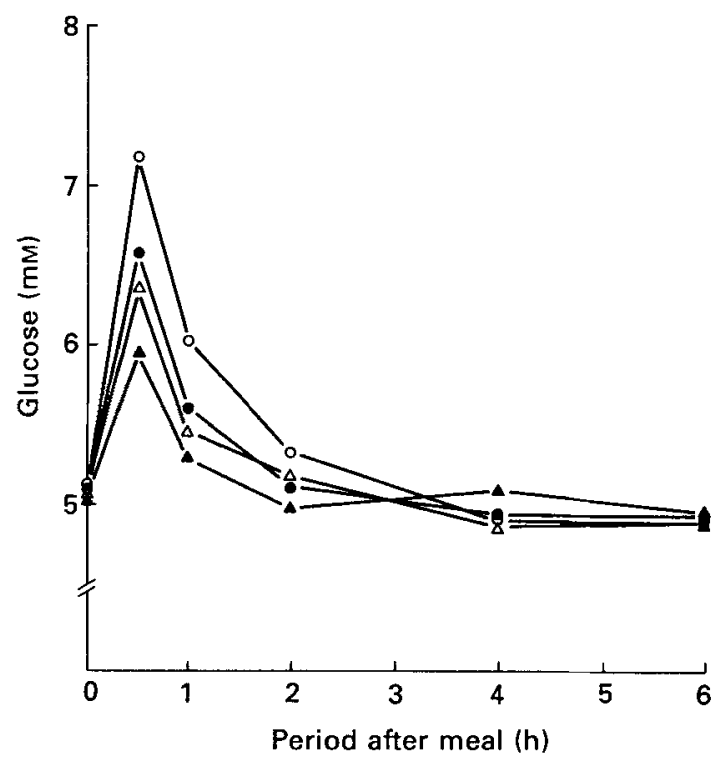

Fig. 1. Mean concentrations ( $n$ 26) of glucose in blood plasma samples from adult male volunteers as a function of the time elapsed after the start of the meal. Carbohydrate content $(\%$ energy):(O), $59 \cdot 3 ;(\mathbf{O})$, $48 \cdot 6 ;(\triangle), 38 \cdot 0 ;(\Delta), 27 \cdot 3$. (For details of meals, see Table 1 and p. 269.)

next measurement ( $60 \mathrm{~min}$ after the start of the meal) was always considerably lower. However, the frequency of blood sampling in the experimental procedure did not permit precise location of the glucose peak; a better location would require shorter intervals. The concentration of glucose at $30 \mathrm{~min}$ was therefore most probably not the highest value occurring postprandially in the blood. Another complicating factor is that the hot meals consisted of two courses, i.e. a hot dish and a dessert, which were consumed in succession. Thus the start of the consumption of the part of the meal containing the largest proportion of sugar, i.e. the dessert, was somewhat variable and depended on the rate at which the main dish was eaten by each participant. Despite these complicating factors, the average of the highest individual glucose values observed may be assumed to correspond with the maximum response to that meal and, therefore, the effects of the compositions of the meals on blood glucose can be compared. A linear relation was found between the average maximum value of glucose and the amount of carbohydrate (Fig. 2): this value increased significantly $(P<0.001)$ on increasing the carbohydrate content in the meal. No significant correlation was found between the height of the postprandial glucose peak and the size of the meals, which were equivalent to $40 \%$ of the mean daily energy intake of each participant according to his dietary history. This result indicated that either the interindividual variations precluded the detection of such a correlation, or to give a participant a meal adjusted to his habitual energy intake instead of a fixed amount of food did not result in a dose-dependent (meal-size-dependent) increase in the height of the postprandial peak.

An alternative way of expressing the postprandial glucose response is the calculation of a glycaemic index (Thorburn et al. 1986; Wolever \& Jenkins, 1986). This has become rather popular and is used by Jenkins et al. $(1983,1984 a, b, 1985,1986)$ in particular. In the procedure used by Wolever \& Jenkins (1986), the peak area of the blood glucose curve after the consumption of a given food component (area KFGHIJ in Fig. 3) is expressed as a percentage of the peak area obtained after the consumption of an equal amount (e.g. $50 \mathrm{~g}$ ) 


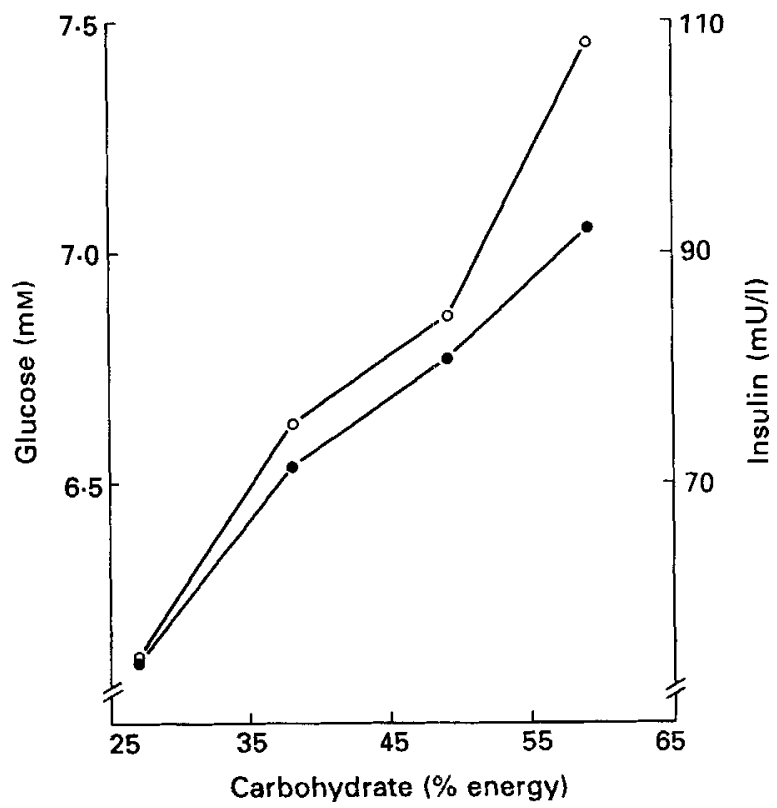

Fig. 2. Averages of the highest concentrations of glucose $(\mathrm{mm})(\mathrm{O})$ and insulin $(\mathrm{mU} / \mathrm{l})(\mathrm{O})(n \mathrm{n} 26)$ as a function of the percentage of energy from carbohydrate in the meals for adult male volunteers. (For details of meals, see Table $I$ and p. 269.)

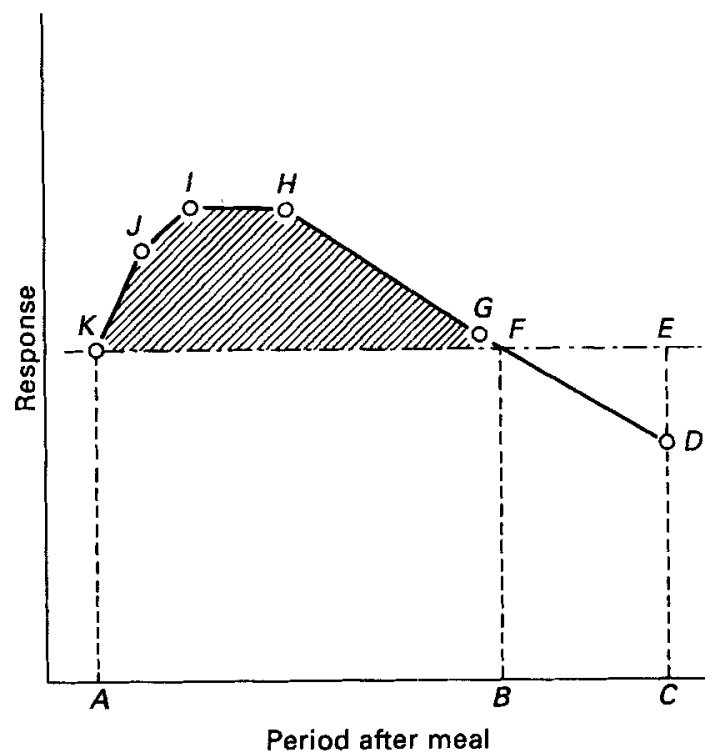

Fig. 3. Schematic representation of the procedure to calculate the glycaemic index. Measured values: $K$, $J, I, H, G, D$; starting value before meal: $K$; full experimental period: $A-C$. 


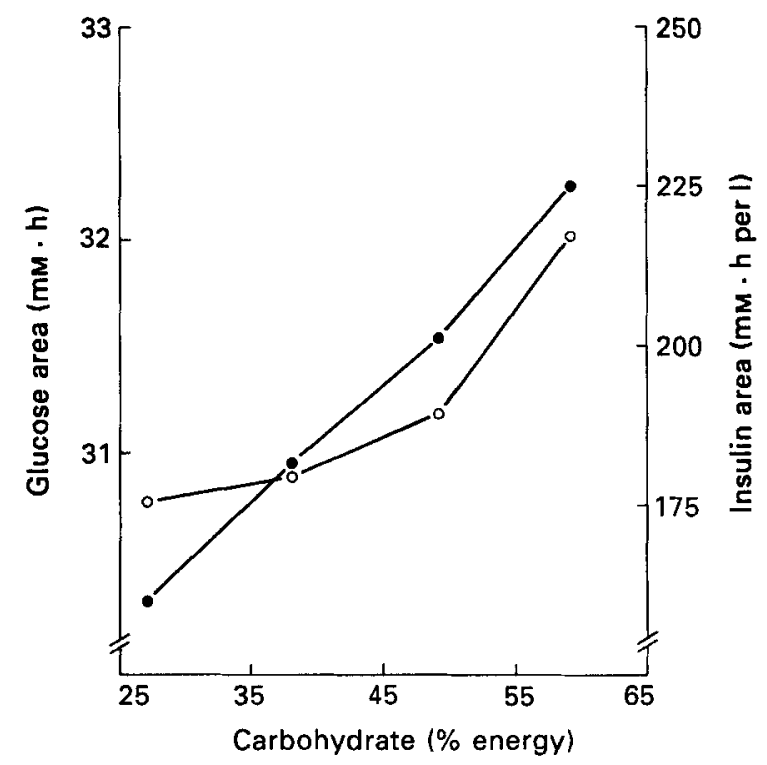

Fig. 4. Mean peak areas $(n$ 26) of the postprandial $(0-6 \mathrm{~h})$ curves of glucose $(\mathrm{mm} \cdot \mathrm{h})(\mathrm{O})$ and insulin $(\mathrm{mU} \cdot \mathrm{h}$ per l) (O) at various carbohydrate contents in the meals for adult male volunteers. (For details of meals, see Table 1 and p. 269.)

of a standard food, i.e. white bread. The food item to be analysed is consumed in the morning after an overnight fast. This approach has been used to establish the potential role of various food components with a view to controlling the blood glucose level in diabetics. However, this procedure has some drawbacks. The final result of the calculation of the area of the postprandial peak (Fig. 3) is highly dependent on the reliability of the determination of the initial glucose concentration $(K)$. In addition, the exact location of the intersection of the curve with the horizontal line through $K$ (point $F$ ) is difficult to establish since the slope in this region is very flat; moreover, the curve may even dip below the initial value $(K)$ depending on the starvation level of the subject before the meal. Since healthy individuals were studied at noon under non-fasting conditions, and in view of the considerations mentioned previously, the postprandial response was calculated as the total peak area under the curve $(A B C D F G H I J K)$. Also the peak areas under the glucose curves appeared to increase significantly $(P<0.05)$ with increasing carbohydrate content (Fig. 4).

\section{Insulin}

The postprandial curves of insulin in the serums are shown in Fig. 5. Analogously to the glucose curves, the values before the meals were rather comparable. Contrary to the glucose curves (cf. Fig. 1), however, the maximum value of insulin occurred later and was on average found in the blood samples taken $60 \mathrm{~min}$ after the start of the meals. The only exception was the meal with the lowest carbohydrate content: here the maximum insulin level was observed $30 \mathrm{~min}$ after the start of the meal. Plotting the maximum insulin concentration as a function of the carbohydrate content of the meals (Fig. 2) showed a strong positive relation between these two variables $(P<0.001)$.

In addition, a strong positive relation $(P<0.001)$ was observed between the peak areas under the insulin curves and the carbohydrate content in the meals (Fig. 4). This indicates 


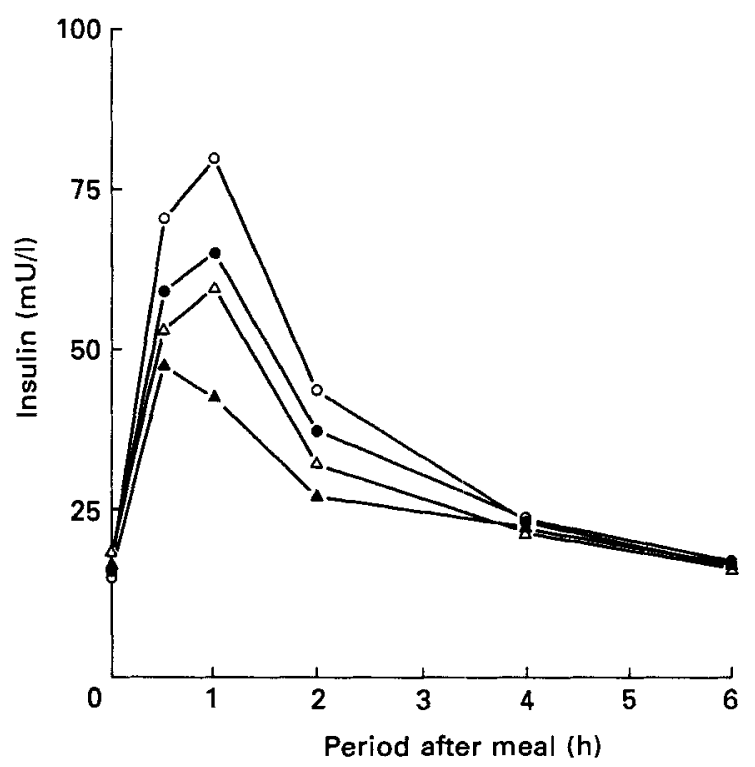

Fig. 5. Mean concentrations ( $n$ 26) of insulin in blood serum samples from adult male volunteers as a function of the time elapsed after the start of the meal. Carbohydrate content (\% energy): (O), 59.3; $(\mathbf{O}), 48 \cdot 6 ;(\triangle), 38 \cdot 0 ;(\Delta), 27 \cdot 3$. (For details of meals, see Table 1 and p. 269.)

that the total amount of insulin secreted after consumption of a meal is larger for carbohydrate-rich meals.

\section{Triacylglycerol and $F G$}

The postprandial curves of TG and FG in the blood samples are represented in Figs. 6 and 7 respectively. Since FG is only a minor fraction (approximately $5 \%$ ) of the TG content in the blood, the TG value may be regarded as a good estimation of triacylglycerol in the blood. The TG curves (Fig. 6) all showed a similar, rapid rise after the consumption of the meals, and the maximum values were found on average at the $2 \mathrm{~h}$ point except for the meal with the lowest fat content where the maximum was on average at the $1 \mathrm{~h}$ point. The maximum postprandial values differed for the four meal types. The highest level was reached after the meals with low $C: F$. The slopes of the curves after the maximum were similar.

These results indicated a similar rate of triacylglycerol metabolism or a similar rate of uptake in the intestine, or both, after the four meals. As a result, there was a much longer period of elevated triacylglycerol levels in the blood after the fat-rich meals. The postprandial curves of FG (Fig. 7) all showed a small and rapid drop after the start of the meals. This reduction in FG level was probably due to decreased lipolysis in adipose tissue after the meal. At $1 \mathrm{~h}$, the FG rose again after the fat-rich meals, apparently due to the release of glycerol from the triacylglycerols circulating in the blood. FG dropped somewhat further after the carbohydrate-rich meals.

Evidently, the rate of glycerol release was reduced because both the plasma triacylglycerol levels in this group were low, and the lipolysis in the adipose tissue was low due to a continued glucose release from complex carbohydrates. For both TG and FG a highly significant $(P<0.001)$ negative linear relation was found between the carbohydrate content of the meals and the peak areas under their postprandial curves (Fig. 8). 


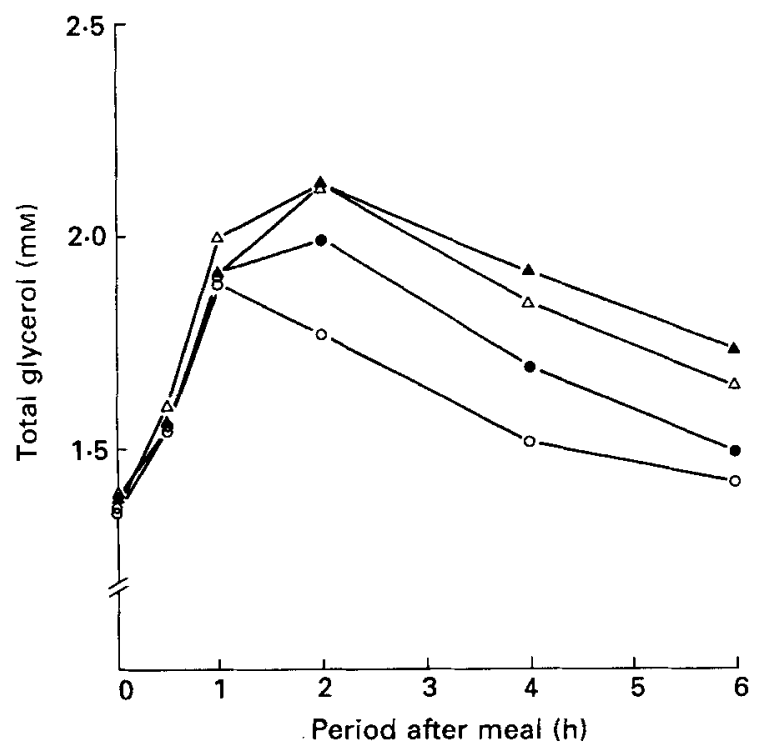

Fig. 6. Mean concentrations ( $n$ 26) of total glycerol in blood serum samples from adult male volunteers as a function of the time elapsed after the start of the meal. Carbohydrate content (\% energy): (O), 59.3; $(\triangle), 48.6 ;(\triangle), 38 \cdot 0 ;(\Delta), 27 \cdot 3$. (For details of meals, see Table 1 and p. 269.)

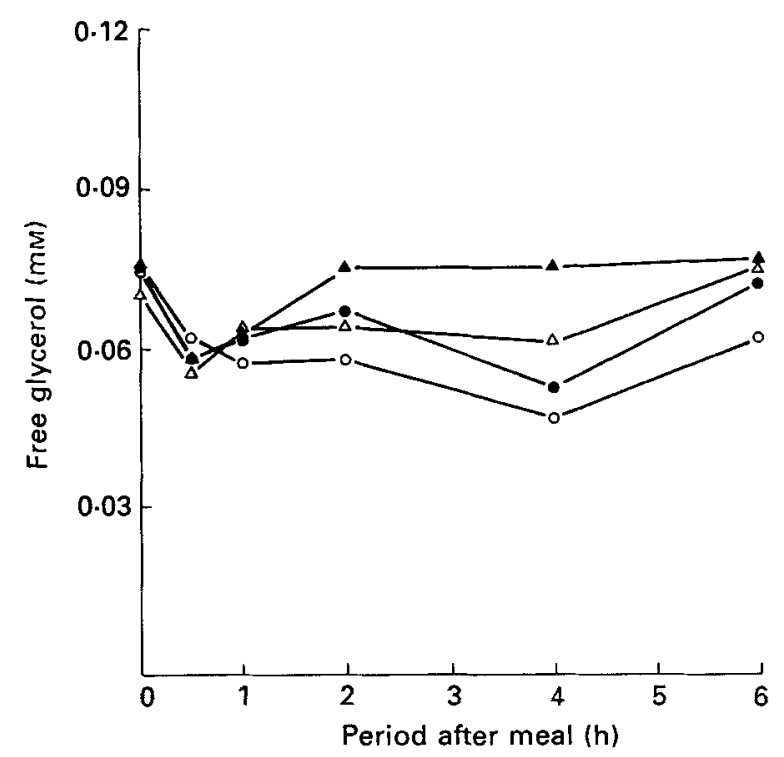

Fig. 7. Mean concentrations ( $n$ 26) of free glycerol in blood serum samples from adult male volunteers as a function of the time elapsed after the start of the meal. Carbohydrate content (\% energy): (O), 59.3; $(\bigcirc), 48 \cdot 6 ;(\triangle), 38 \cdot 0 ;(\Delta), 27 \cdot 3$. (For details of meals, see Table 1 and p. 269.) 

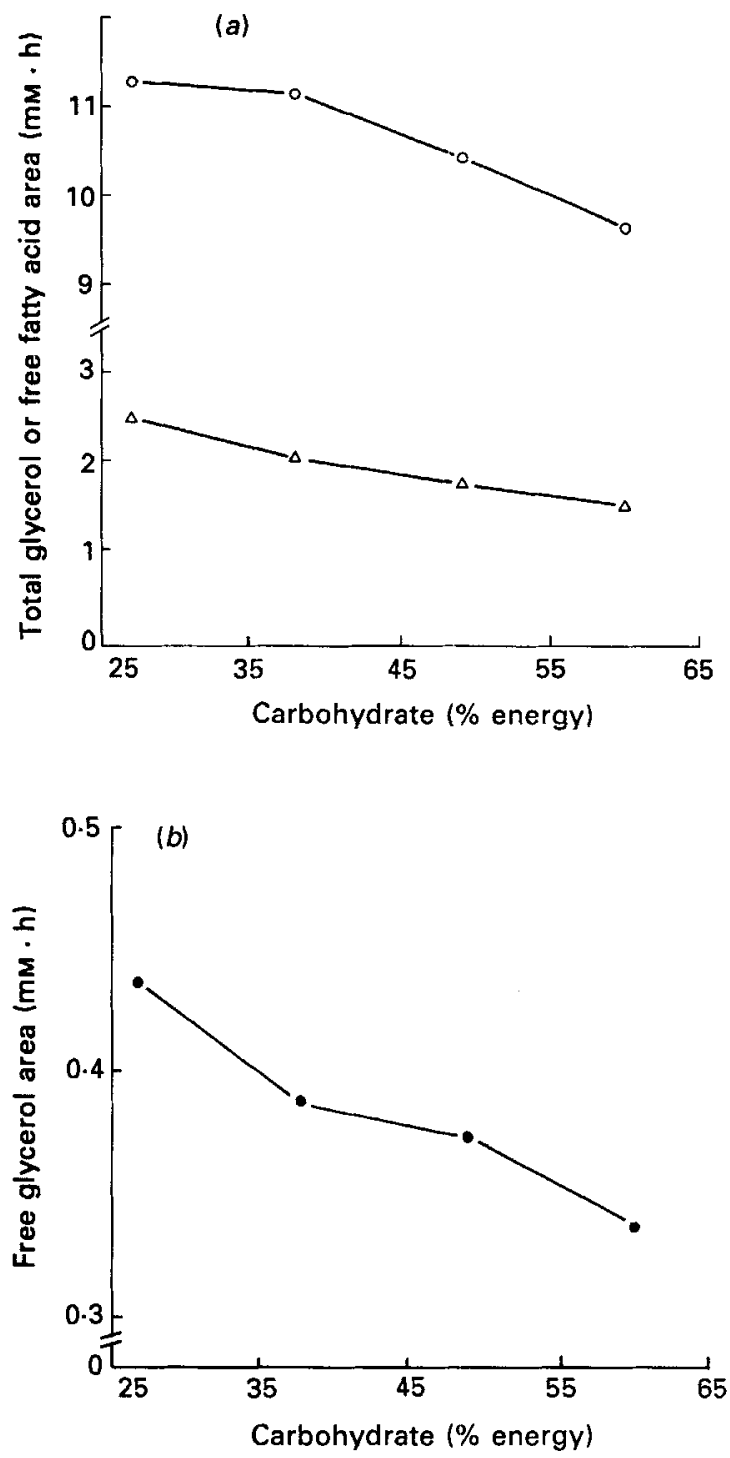

Fig. 8. Mean peak areas $(\mathrm{mM} \cdot \mathrm{h} ; n$ 26) of the postprandial $(0-6 \mathrm{~h})$ curves of $(a)$ total glycerol $(\mathrm{O}),(b)$ free glycerol $(\bigcirc)$ and $(a)$ free fatty acid $(\triangle)$ as a function of the carbohydrate content in the meals given to adult male volunteers. (For details of meals, see Table 1 and p. 269.)

\section{FFA}

The patterns of FFA concentrations in the blood after the four meals and those of FG showed many similarities (Fig. 9). Thus, after an initial rapid drop shortly after the start of the meals, FFA and FG concentration showed a rise after the fat-rich meals, and a further drop after the carbohydrate-rich meals. This similarity was to be expected since in the fasted state both are released from adipose tissue by lipolysis, which is suppressed by carbohydrate feeding, and are liberated from triacylglycerols by lipoprotein lipase $(E C$ 3.1.1.34), particularly after the strong rise in TG following a fat-rich meal. The peak area 


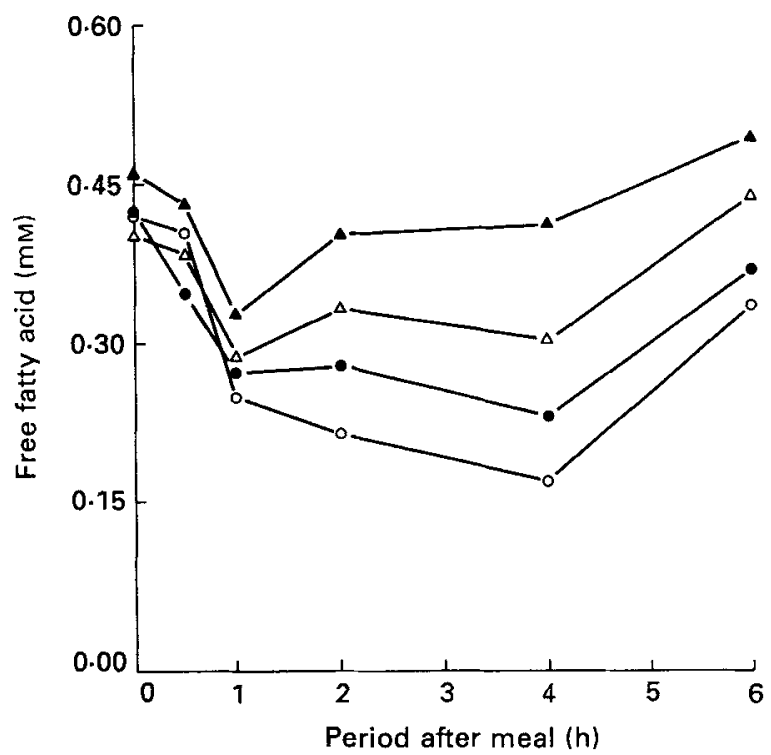

Fig. 9. Mean concentrations ( $n$ 26) of free fatty acid in blood serum samples from adult male volunteers as a function of the time elapsed after the start of the meal. Carbohydrate content ( $\%$ energy):(O), 59.3; $(\mathrm{O}), 48 \cdot 6 ;(\triangle), 38.0 ;(\Delta), 27 \cdot 3$. (For details of meals, see Table 1 and p. 269.)

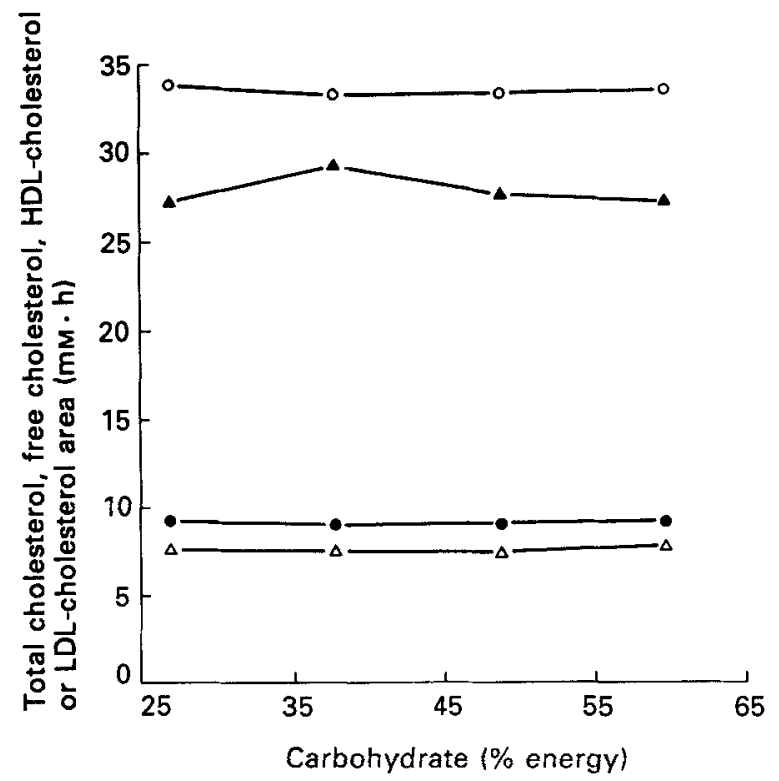

Fig. 10. Mean peak areas $(\mathrm{mm} \cdot \mathrm{h})$ of the postprandial $(0-6 \mathrm{~h})$ curves of total cholesterol $(n 26)(0)$, free cholesterol ( $n$ 26) (O), high-density-lipoprotein (HDL)-cholesterol ( $n$ 12) ( $\triangle$ ), and low-densitylipoprotein (LDL)-cholesterol $(n$ 12) $(\mathbf{A})$ as a function of the carbohydrate content in the meals. (For details of meals, see Table 1 and p. 269.) 
of FFA displayed a highly significant $(P<0.001)$ decrease with increasing carbohydrate content of the meal (Fig. 8).

\section{Cholesterol}

The postprandial curves of total cholesterol and free (unesterified) cholesterol in the serums displayed no significant meal-induced effects (Fig. 10). In view of the fact that the amount of cholesterol in the meals (i.e. in the meat) was nearly constant, and that diet-induced effects on blood cholesterol (such as are induced by changing P:S) take several days to become manifest, these results are in line with expectations. It should, however, be realized that cholesterol in the blood is not transported as a single molecule but esterified to a large extent with a fatty acid (mainly linoleic acid or oleic acid) in complexes with other lipids (triacylglycerols, phospholipids) and proteins, i.e. lipoproteins. Therefore, it was decided to investigate whether changes were detectable in the two main carriers of cholesterol, i.e. LDL and HDL. For logistic reasons however, only a limited number of serums were available. As in the case of total and free cholesterol, no significant effects of meal composition were detectable on the postprandial curves of HDL-cholesterol and LDLcholesterol (Fig. 10).

\section{DISCUSSION}

Before the start of the present study a number of general criteria were laid down for the experiments. Unfortunately, these criteria were not fully met. The dietary histories of the volunteers revealed somewhat higher P:S $(0 \cdot 64$, see Table 3$)$ in their diets than are generally found in the diets of Western populations. It was felt, however, that this would be of little importance to the results. A more serious and complicating fact was that a number of volunteers had problems in consuming the full meal. This may be ascribed to the fact that a significant proportion of the volunteers were not used to eating hot meals and $40 \%$ of their mean daily energy intake at noon. Particularly, the considerable increase in meal volume on increasing the $C: F$ caused problems in consuming the full meal, which illustrates the potential opportunity to reduce energy intake by lowering the fat content of the diet and replacing it with carbohydrate (and fibre). However, the reduced tastiness of the low-fat meals induced an increase in the consumption of salt and gravy (Table 5). As in the prevention of hypertension a reduction of salt intake is indicated, it is important to pay particular attention to the palatability of low-fat meals.

$A$ rather unexpected finding was the observation that the volunteers experienced somewhat stronger hunger feelings at $4 \mathrm{~h}$ after the fat-rich meals than after the carbohydrate-rich meals (Table 4). It has been suggested that fatty acids released on the entrance of food into the duodenum cause feelings of satiety. However, the present study has shown that after about $4 \mathrm{~h}$ these effects are overruled by other satiation stimuli related to the meal such as volume, stomach emptying, fibre, complex carbohydrates, etc.

All four meals resulted in elevated postprandial blood triacylglycerol levels. However, the decline in this elevated level, which set in 1-2 $\mathrm{h}$ after the start of the meals, took much longer with the meals with the lower $\mathrm{C}: \mathrm{F}$. Elevated triacylglycerol levels in the blood have been marked as a potential risk factor for atherosclerosis. Recent findings have indicated that this conclusion may be oversimplified, inasmuch as the extent of the risk may depend on the type of lipoprotein in which the triacylglycerols are incorporated. However, although the risk from the various types of lipoproteins in the blood is not yet fully understood, a potential health risk from an increase in the carbohydrate content of the meal, as will be discussed later, may at least partially be balanced by a decrease in risk due to a lower average postprandial blood triacylglycerol concentration. As expected, the $C: F$ in the meal 
had little or no effect on the postprandial concentrations of total cholesterol, free unesterified cholesterol, HDL-cholesterol and LDL-cholesterol in the blood. The concentrations of FG and FFA reached lower postprandial levels after the meals with the higher $\mathrm{C}: \mathrm{F}$. This is probably due to inhibition of lipolysis in adipose tissue concomitant with a low release from plasma triacylglycerol as a result of the limited postprandial rise of the latter.

The blood glucose curves (Fig. 1) showed considerable postprandial glucose peaks. Moreover, a linear relation was apparent between the amount of carbohydrate in a meal and the height of the peak (Fig. 2). In addition, the peak areas under the glucose curves increased with increasing carbohydrate content (Fig. 4). However, no significant correlation was detected between the amounts of food consumed by the volunteers and the height of the relevant postprandial glucose peaks. These results do not prove that there is no such correlation as there were a number of confounding factors (e.g. variation in body-weight, age, state of health, degree of obesity) which may preclude such an observation. Although the occurrence of a postprandial glucose peak in the volunteers does not seem surprising, it should be realized that frequent high rises in blood glucose are considered undesirable. For diabetics, who either do not produce the glucoregulatory hormone insulin (Type 1 diabetics) or are less responsive to insulin (Type 2 or maturity-onset diabetics), the feasibility is being studied of controlling blood glucose levels by incorporatng slowreleasing complex carbohydrates in the diet.

Prevention of high peaks in the blood glucose level may also be relevant for healthy, nondiabetic people. Our results show a significant effect of $\mathrm{C}: \mathrm{F}$ in the meal not only on the postprandial blood glucose curve (Figs. 1 and 2), but also on the insulin curve (Figs. 5 and 2). Moreover, the peak areas under both the glucose and the insulin curves increase systematically on increasing the C:F (Fig. 4). This indicates that a larger amount of insulin is secreted by the pancreas on increasing the carbohydrate content in the meal. As the P:MD was kept constant at 2:1 (cf. Table 1), the amounts of complex and simple carbohydrates increased simultaneously. It is not yet clear to what extent the various types of carbohydrate in a complex meal affect the amount of insulin secreted by the pancreas but it has been suggested that elevated mean insulin levels in the blood may in the long run lead to insensitivity towards insulin, i.e. maturity-onset diabetes. To prevent excessive release of insulin, it is therefore important to establish what type of carbohydrate is the most suitable to replace fat in a complex meal.

The authors thank the volunteers for their participation in the study; Professors J. G. A. J. Hautvast, J. H. Koeman and H. K. A. Visser for ethical supervision; Miss B. A. van Doorn (MD) for medical screening of the volunteers; Miss P. Swinkels and Mrs W. M. C. Wolbert-Dubelaar for collecting the dietary histories, calculation of the recipes and preparation of the meals; Mr J. Zaalberg for statistical analysis; Messrs C. G. Blonk, A. van der Beek and E. Haddeman for taking the blood samples; and Messrs W. J. Kloots, P. Moret, J. A. Don, J. J. Stam, W. G. L. van Nielen, A. J. van der Kooij and Miss E. M. C. ter Horst for processing the blood samples and performing the various analyses.

\section{REFERENCES}

Calle-Pascual, A. L., Bordiu, E., Romeo, S., Romero, C., Martin-Alvarez, P. J. \& Maranes, J. P. (1986). Food glycaemic index or meal glycaemic response? Human Nutrition, Applied Nutrition 40A, 282-286.

Commissie UCV (1984). Uitgebreide Voedingsmiddelentabel 1984. 's-Gravenhage: Voorlichtingsbureau voor de Voeding. 
Jenkins, D. J. A., Wolever, Th. M. S., Jenkins, A. L., Giordano, C., Giudici, S., Thompson, L. U., Kalmusky, J., Josse, R. G. \& Wong, G. S. (1986). Low glycemic response to traditionally processed wheat and rye products: bulgur and pumpernickel bread. American Journal of Clinical Nutrition 43, 516-520.

Jenkins, D. J. A., Wolever, Th. M. S., Jenkins, A. L., Josse, R. G. \& Wong, G. S. (1984a). The glycaemic response to carbohydrate foods. Lancet ii, 388-391.

Jenkins, D. J. A., Wolever, Th. M. S., Jenkins, A. L., Thorne, M. J., Lee, R., Kalmusky, J., Reichert, R. \& Wong, G. S. (1983). The glycaemic index of foods tested in diabetic patients: a new basis for carbohydrate exhange favouring the use of legumes. Diabetologia 24, 257-264.

Jenkins, D. J. A., Wolever, Th. M. S., Kalmusky, J., Giudici, S., Giordano, C., Wong, G. S., Bird, J. N., Patten, R., Hall, M., Buckley, G. \& Little, J. A. (1985). Low glycemic index carbohydrate foods in the management of hyperlipidemia. American Journal of Clinical Nutrition 42, 604-617.

Jenkins, D. J. A., Wolever, Th. M. S., Wong, G. S., Patten, R., Hall, M., Bird, J., Josse, R. G., Jepson, E. M. \& Little, J. A. (1984 b). Glycemic index of foods: controlling the rate of nutrient absorption in the management of diabetes and hyperlipidemia. In Diet, Diabetes, and Atherosclerosis, pp. 227-239. [G. Pozza, P. Micossi, A. L. Catapano and R. Paoletti, editors]. New York: Raven Press.

Krotkiewski, M. (1984). Effect of guar gum on body-weight, hunger ratings and metabolism in obese subjects. British Journal of Nutrition 52, 97-105.

Marr, J. W. (1971). Individual dietary surveys, purposes and methods. World Review of Nutrition and Dietetics 13 , $105-164$.

Shively, C. A., Apgar, J. L. \& Tarka, S. M. Jr. (1986). Postprandial glucose and insulin responses to various snacks of equivalent carbohydrate content in normal subjects. American Journal of Clinical Nutrition 43, $335-342$.

Thorburn, A. W., Brand, J. C. \& Truswell, A. S. (1986). The glycaemic index of foods. Medical Journal of Australia 144, 580-582.

Wolever, Th. M. S. \& Jenkins, D. J. A. (1986). The use of the glycemic index in predicting the blood glucose response to mixed meals. American Journal of Clinical Nutrition 43, 167-172.

Zilversmit, D. B. (1979). Atherosclerosis: a postprandial phenomenon. Circulation 60, 473-485.

Zilversmit, D. B. (1984). Postprandial hyperlipidemia and its relation to atherosclerosis. In Latent Dyslipoproteinemia and Atherosclerosis, pp. 1-8 [J. L. de Gennes, J. Polonovski and R. Paoletti, editors]. New York: Raven Press. 